\title{
Central Zone of Prostate
}

National Cancer Institute

\section{Source}

National Cancer Institute. CentralZone of Prostate. NCI Thesaurus. Code C128572.

This region of the prostate gland that is located between the peripheral and transition zones, surrounding the ejaculatory ducts. It gives rise to a very small percentage of prostate cancers, though these cancers are frequently more aggressive, often resulting in invasion of the seminal vesicles. 